\title{
'Treat-to-target' with allopurinol is feasible in patients with gout
}

Use of allopurinol doses above those recommended based on creatinine clearance rates can safely and effectively lower serum urate levels in patients with gout-even in patients with renal impairment-report Lisa Stamp and colleagues in a study published in Arthritis \& Rheumatism.

Sustained reduction of serum urate levels to $<360 \mu \mathrm{mol} / \mathrm{l}$ is critical for alleviating the clinical manifestations of gout. In many patients, however, creatinine-clearancebased dosing of allopurinol (the most popular urate-lowering agent) fails to achieve this target. Such dosing aims to prevent potentially fatal allopurinol hypersensitivity syndrome.

The researchers studied allopurinol dose escalation in 45 patients with gout who had serum urate levels $>360 \mu \mathrm{mol} / 1$ despite taking at least the recommended dose of allopurinol for $\geq 1$ month. Allopurinol doses were increased by
50-100 mg per month until adverse events occurred or target serum urate levels $(<360 \mu \mathrm{mol} / \mathrm{l})$ were reached. Of 35 patients, 31 who completed the study had achieved this target after 12 months. Interestingly, the 18 patients receiving furosemide, a loop diuretic, all reached the target, but required higher doses of allopurinol than those not receiving furosemide. No serious adverse events related to allopurinol were reported.

"Larger studies are now required, particularly to address less common side effects and to reinforce our findings on the optimum use of allopurinol in the management of gout," concludes Stamp.

\section{Shreeya Nanda}

Original article Stamp, L. K. et al. Using allopurinol above the dose based on creatinine clearance is effective and safe in chronic gout, including in those with renal impairment. Arthritis Rheum. doi:10.1002/art.30119 